\title{
METODOLOGIA PARA ANÁLISE DE DANOS AMBIENTAIS DO ROMPIMENTO DA BARRAGEM DE FUNDÃO EM BENTO RODRIGUES (MG)
}

\author{
T. R. OLIVEIRA ${ }^{1 *}$, V. S. de OLIVEIRA ${ }^{2}$, M. A. PONTES ${ }^{3}$, M. P. LIBÓRIO ${ }^{4}$, R. M. HADAD 5 , S. LAUDARES \\ Ampla Engenharia e Meio Ambiente ${ }^{1-3}$, Pontifícia Universidade Católica de Minas Gerais ${ }^{1-6}$ \\ thamyresroliveira@gmail.com*
}

Artigo submetido em 06/082017 e aceito em 03/11/2019

DOI: $10.15628 /$ holos.2019.6187

\section{RESUMO}

Em novembro de 2015 ocorreu um dos maiores desastres ambientais já registrados em Minas Gerais e no Brasil, o rompimento da barragem de rejeitos de Fundão. Neste estudo, o objetivo é mensurar a área atingida mais próxima ao rompimento da barragem para quantificar os impactos gerados ao meio ambiente. Para isso, foram utilizadas ferramentas, técnicas e softwares de geoprocessamento para georreferenciar, tratar, classificar e analisar imagens na região do desastre em diferentes períodos. A metodologia aplicada permite medir a área do impacto e consequentemente avaliar os danos ambientais causados pelo desastre não natural. Com base na área de projeto deste estudo, a lama de
\end{abstract}

rejeitos atingiu uma área aproximada de 944 ha, sendo 249,29 ha de vegetação da Mata Atlântica. Afetou também 125,60 ha de uso alternativo do solo. Em relação aos recursos hídricos, foi afetado um total de 21,80 km de cursos d'água, sendo eles: Córrego Santarém, Córrego Vermelho, Rio Gualaxo do Norte, Córrego Mirandinha, Córrego Capião e Córrego Camargo. O uso do geoprocessamento neste estudo atendeu o objetivo proposto, pois com esta tecnologia foi possível mensurar alguns dos danos causados ao meio ambiente após o rompimento da barragem de rejeitos em Bento Rodrigues utilizando imagens de satélite.

PALAVRAS-CHAVE: Desastre Ambiental, Sensoriamento Remoto, Geoprocessamento.

\section{ENVIRONMENTAL DAMAGE ANALYSIS METHODOLOGY OF THE “FUNDÃO" DAM RUPTURE IN BENTO RODRIGUES CITY (MG), BRAZIL}

\begin{abstract}
In November 2015 it happened one of the largest environmental disasters ever recorded in Brazil. The breaking of the tailings dam named "Fundão" in Minas Gerais state. In this study, the goal is to measure the affected area closest to the dam break in order to quantify the impacts to the environment. To accomplish this, it were used tools, techniques and geoprocessing software to geotag, treat, sort, and analyze images of the disaster area at different times. The methodology used enable the measurement of the impact area and consequently assess the environmental damage caused by the natural disaster. Based on the project study area,
\end{abstract}

the tailings slurry reached an area of approximately 944 ha and 249.29 ha of Atlantic Forest vegetation. It also affected 125.60 ha of alternative land use. In relation to water resources, a total of $21.80 \mathrm{~km}$ of watercourses were affected, namely: Santarém Stream, Red Stream, North Gualaxo River, Mirandinha Stream, Capião Stream and Camargo Stream. The use of Geographic Information System - GIS in this study met the proposed objective, because with this technology it was possible to measure part of the damage caused to the environment using satellite images.

KEYWORDS: Environmental Damage, Remote Sensing, Geoprocessing. 


\section{INTRODUÇÃO}

Em novembro de 2015, Jardim (2016) relata que ocorreu um dos maiores desastres ao meio ambiente já registrado em Minas Gerais e no Brasil. O rompimento da barragem de rejeitos da mineradora Samarco, em Mariana, provocou, conforme relatório do IBAMA - Instituto Brasileiro do Meio Ambiente e dos Recursos Naturais Renováveis (2015), danos ao meio ambiente, à economia e a sociedade, numa extensão de $663,2 \mathrm{Km}$. A extensa lama de rejeitos, como afirmam Pinto et al. (2015), atingiu a bacia hidrográfica do Rio Doce e parte dos 228 municípios da bacia no estado de Minas Gerais e do Espírito Santo.

A literatura apresenta metodologias que utilizam o geoprocessamento em estudos de degradação ambiental e análises de riscos de desastres como mostram os trabalhos de Medina (1994), Maskrey (1998), Barbosa et al. (2007), Sousa et al. (2007), Sousa et al. (2008) e Carvalho et al. (2011). O geoprocessamento, para Costa Lima (1999) aplicado ao processo resultante da interação ambiente e sociedade, dinamiza a geração de informações, eficientiza a produtividade por meio de atualizações em tempo real e versatiliza o manuseio dos dados.

A resposta para o problema do cálculo do dano ambiental passa pela avaliação e mensuração das mudanças ocorridas em áreas atingidas pelo desastre em Bento RodriguesMariana-MG, através da manipulação de imagens de satélite utilizando softwares dedicados exclusivamente para o tratamento de imagens. Através destes softwares é possível obter imagens com diferentes composições de cores e obter informações necessárias e relevantes para a elaboração de mapas temáticos, como mostram Galo et al. (2002). Tal procedimento é bastante utilizado em estudos e monitoramentos de desastres naturais pela sua facilidade e pela maior e melhor visualização da área estudada, além de existirem diversas técnicas para o seu processamento e para a obtenção de informações sobre as imagens como mostram os trabalhos de Moura et al. (2005) e Longhitano (2010).

A mensuração do alcance, profundidade e temporalidade do desastre é um problema composto por múltiplas funções. Neste caso, é importante observar o tamanho da área atingida pelo rejeito mais próxima ao rompimento da barragem para integrar no cálculo dos impactos ao meio ambiente e quantificá-los em função do tempo e do espaço geográfico.

Esta pesquisa demonstra os benefícios gerados pelo uso de Sistemas de Informação Geográfica (SIG) na avaliação de danos ambientais ocasionados por desastres não naturais, neste caso, o rompimento da barragem em Bento Rodrigues-MG. Marcelino et al. (2006), Silva (2009) e Saito et al. (2011) ratificam esses benefícios através de análises ambientais e monitoramentos de desastres naturais, onde se buscam soluções para situações de desastres ambientais ocasionados por ações antrópicas.

\section{SENSORIAMENTO REMOTO APLICADO NA ANÁLISE ESPACIAL DE DESASTRES AMBIENTAIS}

Um dos principais objetivos do sensoriamento remoto é a aquisição de informações sobre a superfície de terra para mapeamento e avaliação de recursos terrestres e monitoramento ambiental, como afirmam Aragão e Almeida (2009) e Meneses e Sano (2012). Estas 
geotecnologias, como destaca Silva (2009), possibilitam a coleta, armazenamento e análise de grande quantidade de dados, que devido à complexidade dos desastres naturais e ambientais, seriam praticamente inviáveis de serem tratados utilizando os tradicionais métodos analógicos.

Inicialmente, considerado como um ramo da fotogrametria, o sensoriamento remoto tem como característica a capacidade de gerar dados dos sistemas orbitais através de sensores de alta resolução que registram imagens periódicas da terra. O processamento dessas imagens oferece, como mostram Pegoraro et al. (2013), diversas possibilidades de interpretação e análises temporais.

Atualmente, a resolução espectral das imagens obtidas pelos sensores imageadores já ultrapassa centenas de bandas. A resolução espacial de muitas imagens já é menor que 1 metro, possibilitando diversas aplicações. Meneses e Almeida (2012) apresentam por exemplo aplicações em levantamentos de recursos naturais e mapeamentos temáticos, monitoração ambiental, detecção de desastres naturais, desmatamentos florestais, previsões de safras, cadastramentos multifinalitários, cartografia de precisão, defesa e vigilância.

O acelerado avanço com que o sensoriamento remoto se desenvolveu em poucas décadas deve-se à revolução nos meios de se observar a terra numa escala global e periódica, e na rapidez da monitoração dos fenômenos dinâmicos e das mudanças das feições terrestres. $O$ sensoriamento remoto é, conforme Meneses e Almeida (2012), uma tecnologia empregada em escala mundial e que, talvez, reúna a maior diversidade de pesquisadores e usuários em torno de uma tecnologia de aplicação.

Em Brasil (1986), o Conselho Nacional do Meio Ambiente - CONAMA, órgão ambiental brasileiro, define o impacto ambiental como qualquer alteração das propriedades físicas, químicas e biológicas do meio ambiente. Sendo esta originada de qualquer forma de matéria ou energia decorrente das atividades humanas que afetam direta ou indiretamente a saúde e o bem-estar da população, a biota, atividades sociais e econômicas, condições estéticas e sanitárias do meio ambiente e a qualidade dos recursos ambientais.

A norma internacional ISO 14.001 de 2004, descrita por Sanchéz (2015), define impacto ambiental como "qualquer modificação do meio ambiente, adversa ou benéfica, que resulte, no todo ou em parte, das atividades, produtos ou serviços de uma organização".

O problema dos impactos ambientais causados pela mineração, foco central da pesquisa de Silva (2007), é apresentado como uma consequência das atividades humanas com fins econômicos. Em particular, a mineração e a agricultura são as duas atividades econômicas básicas da economia mundial que alteram o meio ambiente. Através destas, o homem extrai recursos naturais que alimentam toda a economia. Sem elas, nenhuma das atividades subsequentes pode existir. A mineração, evidentemente, causa um impacto ambiental considerável. Ela altera intensamente a área minerada e as áreas vizinhas, onde são feitos os depósitos de estéril e de rejeito.

Do ponto de vista jurídico, Cerri Neto (2007) expõe que a defesa civil, através do Ministério da Integração Nacional, não define legalmente o dano ambiental. De modo amplo, o dano ambiental é a consequência de um desastre causado pela ação do homem (culposa ou não), lesando o meio ambiente. Este fato pode ser intensificado pelas perdas humanas, materiais ou ambientais induzidas às pessoas, comunidades, instituições, instalações e/ou ecossistemas. De 
outro modo, Bittar Filho (1994) afirma que o dano ambiental não consiste apenas na lesão ao equilíbrio ecológico, afeta igualmente outros valores essenciais da coletividade a ele ligados, como a qualidade de vida a saúde e de forma mais abrangente o contexto moral coletivo, nas bases da atualidade jurídica.

Assim, nas definições de Brasil (1986) e Sanchéz (2015), o impacto ambiental é uma consequência das ações humanas, podendo ser positivas ou negativas. Logo, o rompimento da barragem em Mariana, que provocou diversos impactos ambientais com consequências nos processos naturais (modificação do uso do solo) e socioeconômicos (perdas humanas e materiais da comunidade de Bento Rodrigues), causou danos ao meio ambiente, pois conforme a concepção de dano exposta em Fenker (2011), os impactos negativos superaram os impactos positivos, resultando em prejuízos.

A análise espacial, de acordo com Câmara et al. (2002), é composta por um conjunto de procedimentos, muitos deles encadeados, que têm por objetivo a seleção de informações geográficas de interesse, diante de um domínio de problema, e incluem um conjunto de métodos genéricos de análise exploratória e a visualização dos dados que, em geral, se dá por meio de mapas. Esse ambiente permite descrever a distribuição das variáveis de estudo, identificar observações e buscar a existência de padrões na distribuição espacial. Com esse procedimento é possível elaborar hipóteses e selecionar o modelo que melhor explique os fenômenos espaciais.

Análise espacial é uma ferramenta essencial para estudar e compreender processos ou fenômenos naturais ou de origem antrópica, fornecendo um conjunto de informações que caracteriza o estado e/ou a situação do meio. Logo, a análise espacial estuda as evoluções espacial e temporal desses processos no âmbito geográfico e as suas inter-relações, sendo essas informações importantes em estudos ambientais. Vários trabalhos utilizaram essa ferramenta como instrumento de estudo de fenômenos, tais como Zerger e Smith (2003) e Mora e Keipi (2006).

Assim como no presente trabalho, o geoprocessamento tem sido empregado para medir áreas inundadas em situações de rompimento de barragens como mostram Collischonn e Tucci (1997) e Chaves et al. (2015), bem como Petta et al. (2005) para identificar áreas de risco.

A simulação da ruptura da barragem de Ernestina, no rio Jacuí, Rio Grande do Sul, através da utilização de técnicas de geoprocessamento realizada por Collischonn e Tucci (1997) permitiu medir possíveis consequências, extensões de áreas inundadas e população atingida caso ocorresse o desastre.

Uma simulação de ruptura de barragem foi realizada por Chaves et al. (2015) nas bacias hidrográficas do Ribeirão do Roque e Rio Claro localizado no estado de São Paulo. Nesta simulação, foram analisados os principais critérios utilizados na escolha do modelo hidrodinâmico, do hidrograma de ruptura, bem como realizados o mapeamento das áreas potencialmente inundáveis, que foi elaborado no software ArcGIS (ESRI, 2006).

As técnicas de geoprocessamento em um SIG, permitiram Petta et al. (2005) obter cartas de vulnerabilidade ambiental e natural das áreas exploradas pela PETROBRAS no município de Mossoró em Rio Grande do Norte. O levantamento dos recursos naturais foi obtido através do sensoriamento remoto, além disso, foi desenvolvido um banco de dados geográfico que permitiu a análise espacial das informações geradas, no qual pode ser utilizado como um sistema de 
ferramentas de apoio a decisões de gestão, diagnóstico e ações de controle ambiental nas áreas de risco.

\section{METODOLOGIA}

Este trabalho apresenta uma pesquisa experimental, onde uma metodologia é desenvolvida pela aplicação de técnicas de geoprocessamento, fazendo inferências sobre o desastre ambiental ocorrido em Mariana-MG. Para tanto, foram testadas ferramentas e técnicas em softwares de geoprocessamento para tratar e analisar dados quantitativos e descritivos.

O município de Mariana situa-se na vertente sul da Serra do Espinhaço, na Zona Metalúrgica de Minas Gerais, conhecida como Quadrilátero Ferrífero. A figura 1 representa a localização de Mariana que está a 697 metros de altitude a uma distância aproximada de $12 \mathrm{~km}$ de Ouro Preto e 110 km de Belo Horizonte. Os municípios limítrofes de Mariana são Ouro Preto, Barra Longa, Diogo de Vasconcelos, Acaiaca, Piranga, Catas Altas e Alvinópolis (Mariana, 2016).

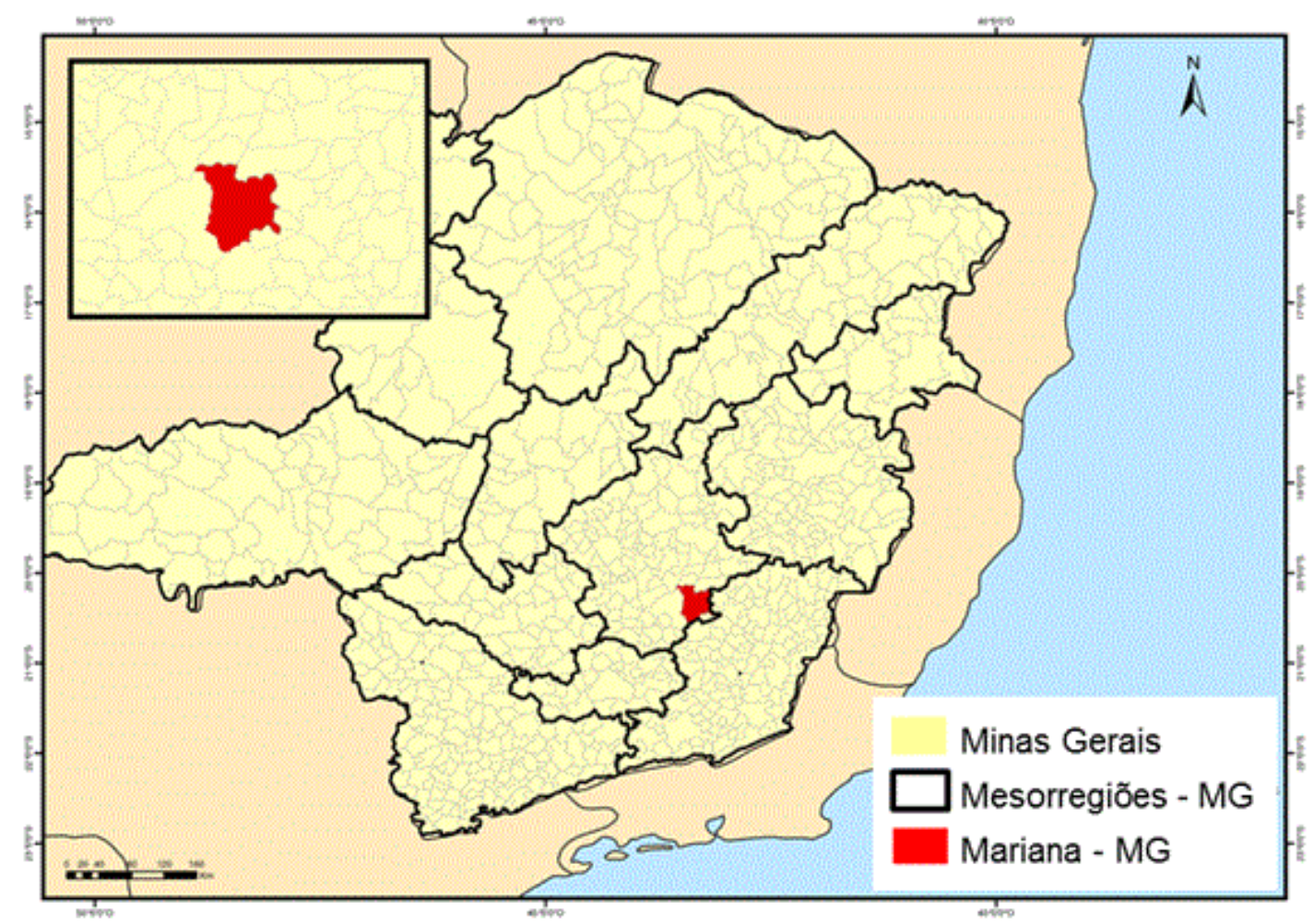

Figura 1: Localização do Município de Mariana. Fonte: Lorenzeto (2006).

A cidade de Mariana possui nove Distritos, um deles é Santa Rita Durão que contempla o subdistrito de Bento Rodrigues localizado a $35 \mathrm{~km}$ da sede, que foi importante centro de mineração do século XVIII, conforme destaca Mariana (2016). Bento Rodrigues foi uma das localidades mais afetada diretamente pelo rompimento da barragem de rejeitos. Portanto, esta é a área de interesse central deste estudo.

A Samarco é a empresa responsável pela Barragem de Fundão, a qual se rompeu em 05 de novembro de 2015. A empresa do setor de mineração, fundada em 1977, é controlada igualmente pelos acionistas BHP Billiton Brasil Ltda. e Vale S.A Samarco (2015). O principal produto gerado são pelotas de minério de ferro que são comercializadas para a indústria 
siderúrgica mundial. Exporta o seu produto para 19 países das Américas, Oriente Médio, Ásia e Europa.

A atividade de mineração, para Fernandes Neto et al. (2015), está associada à produção de resíduos, que requerem tratamento e disposição adequados para não impactarem o ambiente. Na mineração são gerados dois tipos principais de resíduos que são os estéreis, produzidos pela lavra ou retirada do minério da jazida, e os rejeitos, produzidos pelo seu beneficiamento.

No estudo de Araújo (2006) observou-se que muitas empresas de mineração, para reduzir os custos envolvidos na construção das estruturas de contenção de rejeitos, optam pela utilização do próprio rejeito como elemento construtivo sem controle tecnológico, o que em muitos casos já causou graves acidentes devido a ruptura das estruturas. Porém, Pimentel (2016) destaca que o rompimento da barragem de Fundão foi ocasionado pela elevada saturação de rejeitos arenosos depositados na mesma, falhas no monitoramento, equipamentos com defeito, número pequeno de equipamentos de monitoramento, elevada taxa de alteamento anual da barragem, assoreamento do dique e deficiência junto ao sistema de drenagem.

Os softwares utilizados nesta pesquisa foram: o Spring (INPE, 2016), o Regeemy (INPE, 2008) e o ArcGIS (ESRI, 2006). No Spring foram utilizadas as técnicas de restauração, aplicação de contraste, filtragem, composição colorida e classificação supervisionada. No Regeemy foi utilizada a técnica para georreferenciar as imagens automaticamente por algoritmos. No ArcGIS foram utilizadas técnicas para criação de mapas, compilação de dados geográficos, análise de informações mapeadas, gestão de informações geográficas em bancos de dados, classificação supervisionada e confecção dos mapas temáticos. A metodologia aplicada neste estudo, ilustrada na figura 2, é detalhada em 10 etapas.

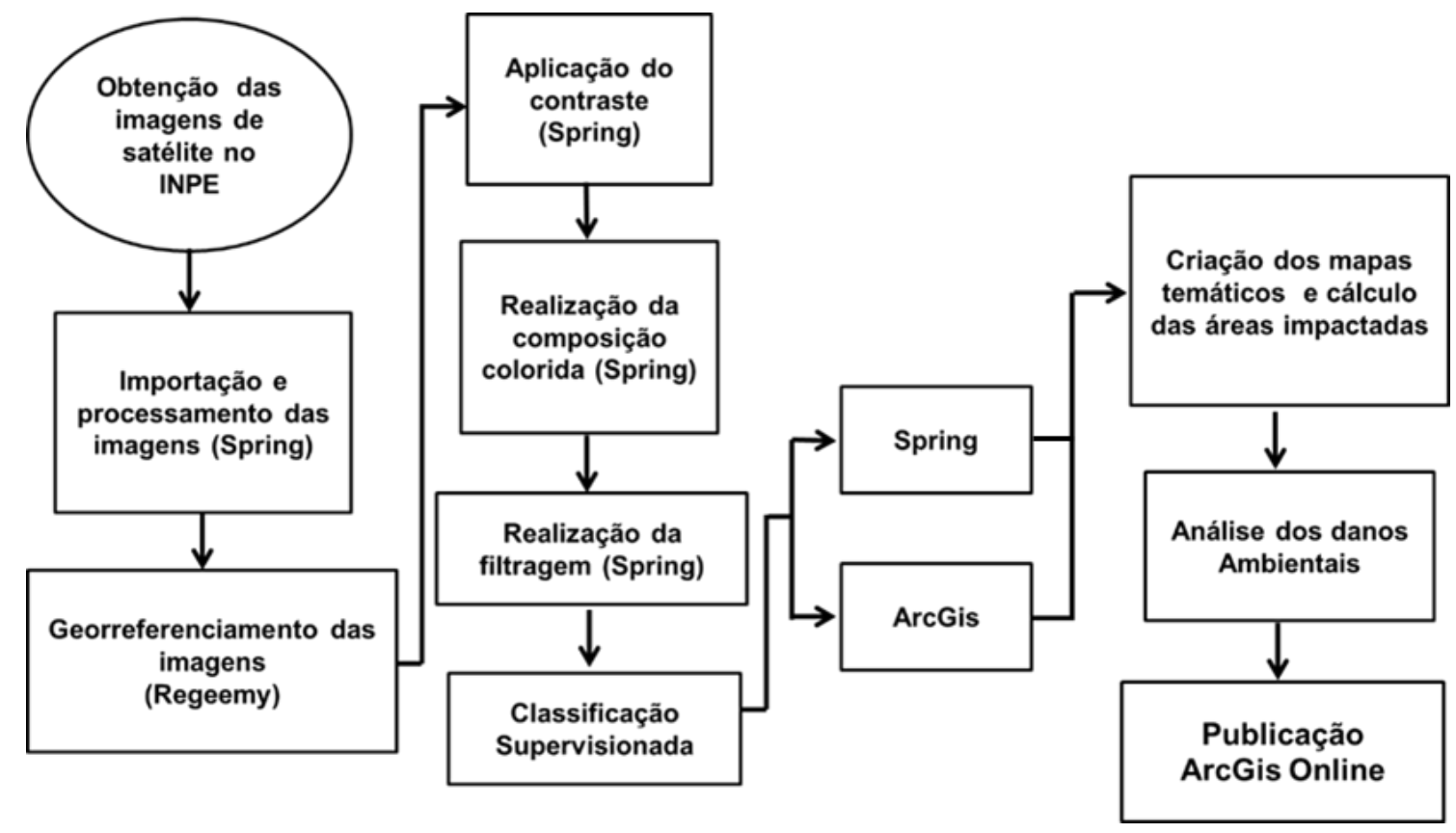

Figura 2: Fluxograma das etapas do experimento. Fonte: autores.

$\mathrm{Na}$ etapa 1 foi feita a obtenção das imagens de satélites do Instituto Nacional de Pesquisas Espaciais - INPE: As imagens obtidas foram do satélite LANDSAT-8, sensor OLI, órbita 28 , ponto 074 , com datas das imagens de 11 de outubro de 2015, sendo esta a imagem de antes 
do rompimento da barragem, e de 12 de novembro de 2015, sendo esta, após o rompimento. Vale ressaltar que foi realizada a pesquisa das imagens em diversas datas, porém as imagens escolhidas foram as que apresentaram a menor quantidade de nuvens, 2,12 e 6,32 \%, respectivamente, o que facilitou a manipulação das imagens e a obtenção de dados mais confiáveis.

$\mathrm{Na}$ etapa 2 foi realizada a importação e processamento das imagens pelo software SPRING. Para facilitar a identificação da área impactada e a análise dos componentes das imagens, foi realizada a importação das imagens em formato GeoTIFF para o software SPRING, com delimitação das coordenadas de projeto: $X 1=656543.00$ e $Y 1=7757188.00$ e X2=670094.00 Y2=7767225.00, coordenadas planas UTM, Datum: WGS-84. Para melhorar a qualidade das imagens foi realizada a restauração das bandas para resolução espacial de 15 metros, utilizando os parâmetros do Landsat 8, sensor OLI, parâmetros estes disponíveis nas versões mais atuais do Software Spring (5.4.0 e 5.4.2).

A etapa 3 consistiu no georreferenciamento das imagens. Após a restauração foi necessário o georreferenciamento das bandas utilizadas. Este procedimento é importante devido ao pequeno deslocamento que ocorre na imagem após o procedimento de restauração. Esta etapa foi realizada no software Regeemy. Foi utilizada a banda 8 como referência para o georreferenciamento das imagens utilizadas neste estudo, por esta banda já ter resolução espacial de 15 metros.

$\mathrm{Na}$ etapa 4 foi realizada a aplicação de contraste. Após o georreferenciamento, aplicou-se o contraste linear nas bandas utilizadas para que as imagens ficassem mais nítidas. A aplicação do contraste, afirma Santos et al. (2010), tem por objetivo modificar os níveis de cinza de uma imagem de forma que algumas informações espectrais sejam destacadas, não aumentando a quantidade de informação, mas melhorando a sua percepção. O contraste aplicado nas bandas utilizadas foi o realce linear. Este procedimento consiste no aumento do espalhamento dos níveis de cinza, conforme uma função linear (IBGE, 2001).

$\mathrm{Na}$ etapa 5, se realizou o procedimento de filtragem. As técnicas de filtragem são transformações da imagem pixel a pixel, que não dependem apenas do nível de cinza de um determinado pixel, mas também do valor dos níveis de cinza dos pixels vizinhos. O processo de filtragem é realizado utilizando matrizes denominadas máscaras, as quais são aplicadas sobre a imagem. Utilizou-se o filtro Linear-Realce TM, que é o filtro utilizado para imagens Landsat.

Após os procedimentos de filtragem, realizou, na etapa 6 , a composição colorida utilizando as bandas 4, 6 e 7, cujos comprimentos de ondas e a finalidade de cada banda estão apresentados no quadro 1 . A composição utilizada neste estudo foi RGB-764, denominado falsacor, sendo a combinação que mais realçou os alvos classificados neste estudo.

Quadro 1: Comprimento de onda das bandas utilizadas do Satélite Landsat 8, sensor OLI.

\begin{tabular}{|l|c|l|}
\hline Bandas & $\begin{array}{c}\text { Comprimento de } \\
\text { onda (micrômetros) }\end{array}$ & \multicolumn{1}{|c|}{ Utilização/mapeamento } \\
\hline 4 Vermelho & $0,64-0,67$ & Discrimina encostas de vegetação. \\
\hline 6 SWIR 1 & $1,57-1,65$ & $\begin{array}{l}\text { Discrimina conteúdo do solo e da vegetação de } \\
\text { umidade; penetra nuvens finas. }\end{array}$ \\
\hline
\end{tabular}




\begin{tabular}{|l|l|l|}
\hline 7 SWIR 2 & $2,11-2,19$ & $\begin{array}{l}\text { Melhorou o conteúdo do solo e da vegetação e } \\
\text { penetração nuvem fina de umidade. }\end{array}$ \\
\hline
\end{tabular}

Fonte: USGS (2016)

$\mathrm{Na}$ etapa 7 foi feita a classificação supervisionada das imagens. A classificação foi realizada através de dois softwares: Spring e ArcGIS. A predefinição das classes para classificação supervisionada foi baseada nos alvos identificados nas imagens e as amostras da classificação foram obtidas com o auxílio de características como cor, textura, tonalidade, entre outros. As classes identificadas foram: povoado, vegetação, mineração, lama, uso alternativo do solo e água. O método classificador utilizado foi o Maxver, que consiste no modelo estatístico de "Máxima Verossimilhança", sendo muito utilizado em trabalhos de mapeamento temático desenvolvidos pelo Spring ou outros Sistemas de Informações Geográficas (SIG) como o ArcGIS. O algoritmo obtém a média dos níveis digitais de cada classe e estabelece uma distribuição de probabilidade normal, e passa a considerar a ponderação das distâncias entre as classes para classificar o pixel de acordo com sua posição em relação à distribuição normal. Em alguns casos, um mesmo pixel pode ser atribuído a duas classes distintas (efeito da sobreposição das distribuições normais das classes), sendo assim, é necessário estabelecer um limiar de aceitação para refinar a classificação conforme seu propósito. Para reduzir o efeito da sobreposição no processo de classificação, conforme cita Cruz et al. (2009), é necessário um conjunto de treinamento supervisionado com uma quantidade significativa de pixels de alvos distintos, permitindo um trabalho estatístico mais eficiente e um resultado mais próximo da realidade. 0 limiar de aceitação utilizado no Spring foi de $100 \%$, ou seja, houve a classificação de todos os pixels das imagens. Após esta classificação foi realizado o refinamento da imagem com base na composição colorida original para aumentar a diferenciação das classes identificadas. Por fim, a metodologia foi replicada por duas vezes, atingindo sempre os mesmos resultados.

Na etapa 8 foi feita a criação dos mapas temáticos e o cálculo das áreas impactadas. Para a estimativa das áreas afetadas pela lama oriunda do rompimento da barragem, utilizou-se o software ArcGIS no qual através de ferramentas disponibilizadas pelo mesmo foi possível sobrepor e extrair informações das áreas impactadas pelo rompimento da barragem. Após a extração desta informação, confeccionou-se o mapa temático.

Na etapa 9 foi feita a análise dos danos ambientais. Através das informações das áreas impactadas foi realizada a análise dos danos, no qual obteve as extensões e quais recursos hídricos foram afetados pela lama, bem como, a mensuração das áreas das classes do mapa temático. A camada de dados que representa a lama foi sobreposta na imagem de antes do rompimento da barragem, permitindo calcular a área atingida pela lama. Em relação aos recursos hídricos, foi utilizado o banco de dados da ANA - Agência Nacional de Águas, para identificar os cursos afetados e obter a extensão de cada curso d'água.

Por fim, na etapa 10, foi feita a publicação dos resultados no ArcGIS online. Após a execução de todas as etapas descritas anteriormente, foi publicado no ArcGIS online parte do produto deste estudo, podendo ser acessado por qualquer pessoa que tenha cadastro no site. $O$ mapa gerado é interativo e várias pessoas podem acessá-lo simultaneamente. 


\section{RESULTADOS E DISCUSSÕES}

O processamento da imagem ocorreu conforme as etapas demonstradas na metodologia. Ao utilizar a composição colorida RGB-764, obteve-se o resultado conforme demonstrado nas Figura 3 (a) e (b). A composição colorida destacou os elementos presentes na área de estudo, tais como: mineração, lama, cobertura vegetal, água, povoado e áreas de uso alternativo do solo.

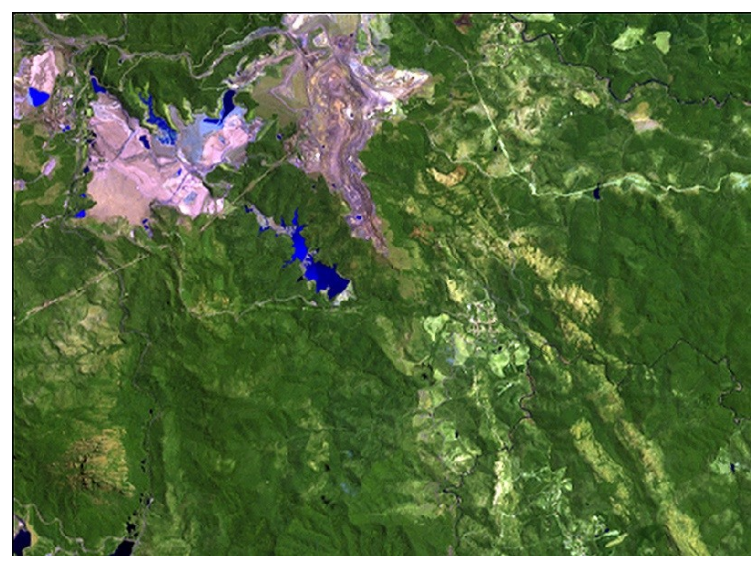

(a)

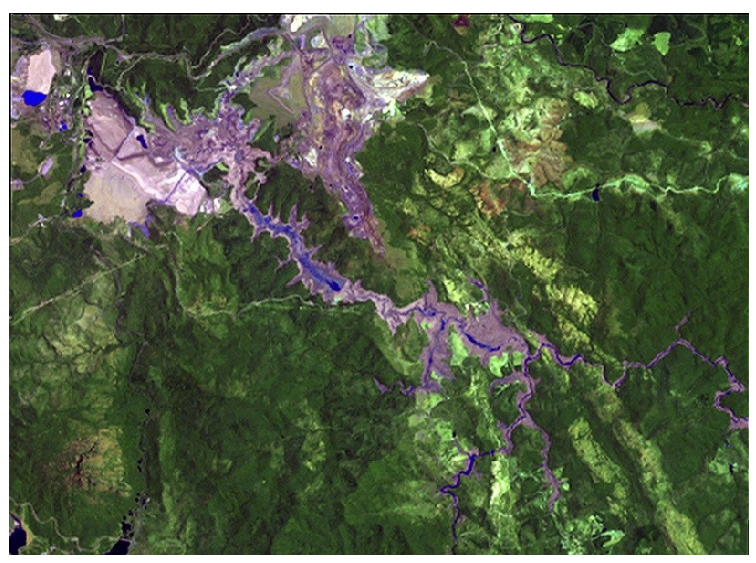

(b)

Figura 3: Composição colorida (a) antes e (b) após o rompimento da barragem. Fonte: autores.

Com base nas imagens coloridas apresentadas, realizou-se a classificação das imagens. Observa-se nas Figuras 4 e 5 os resultados das classificações realizadas no Spring.

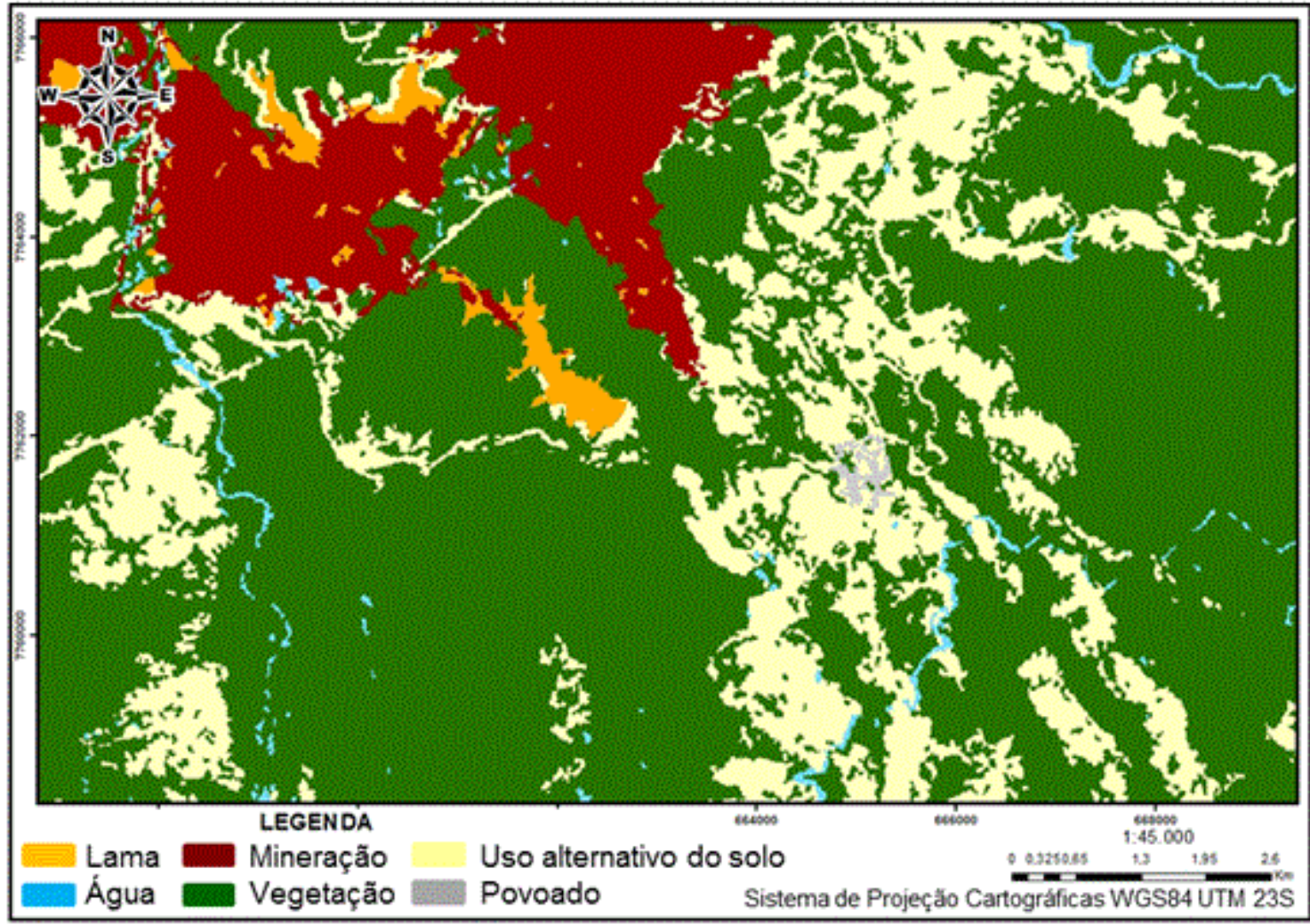

Figura 4: Classificação uso do solo pelo Spring antes do desastre. Fonte: autores. 


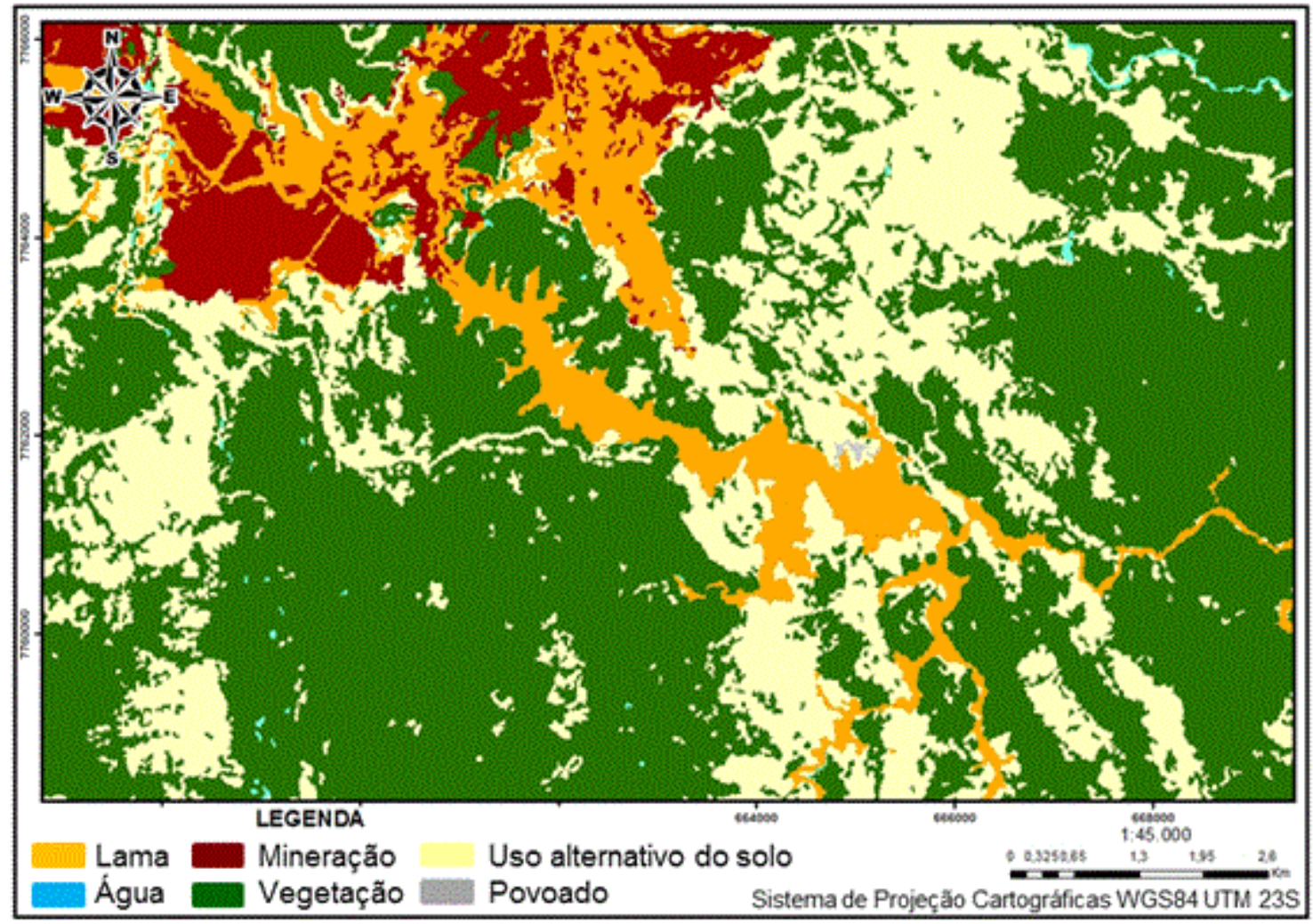

Figura 5: Classificação uso do solo pelo Spring após o desastre. Fonte: autores.

Nas Figuras 6 e 7 são apresentadas as classificações realizadas no ArcGIS. Dentre as classes obtidas, a classe de uso alternativo do solo compreende as áreas de cultivo, áreas de pastagem, além de estradas e vias de acesso identificado nas imagens estudadas.

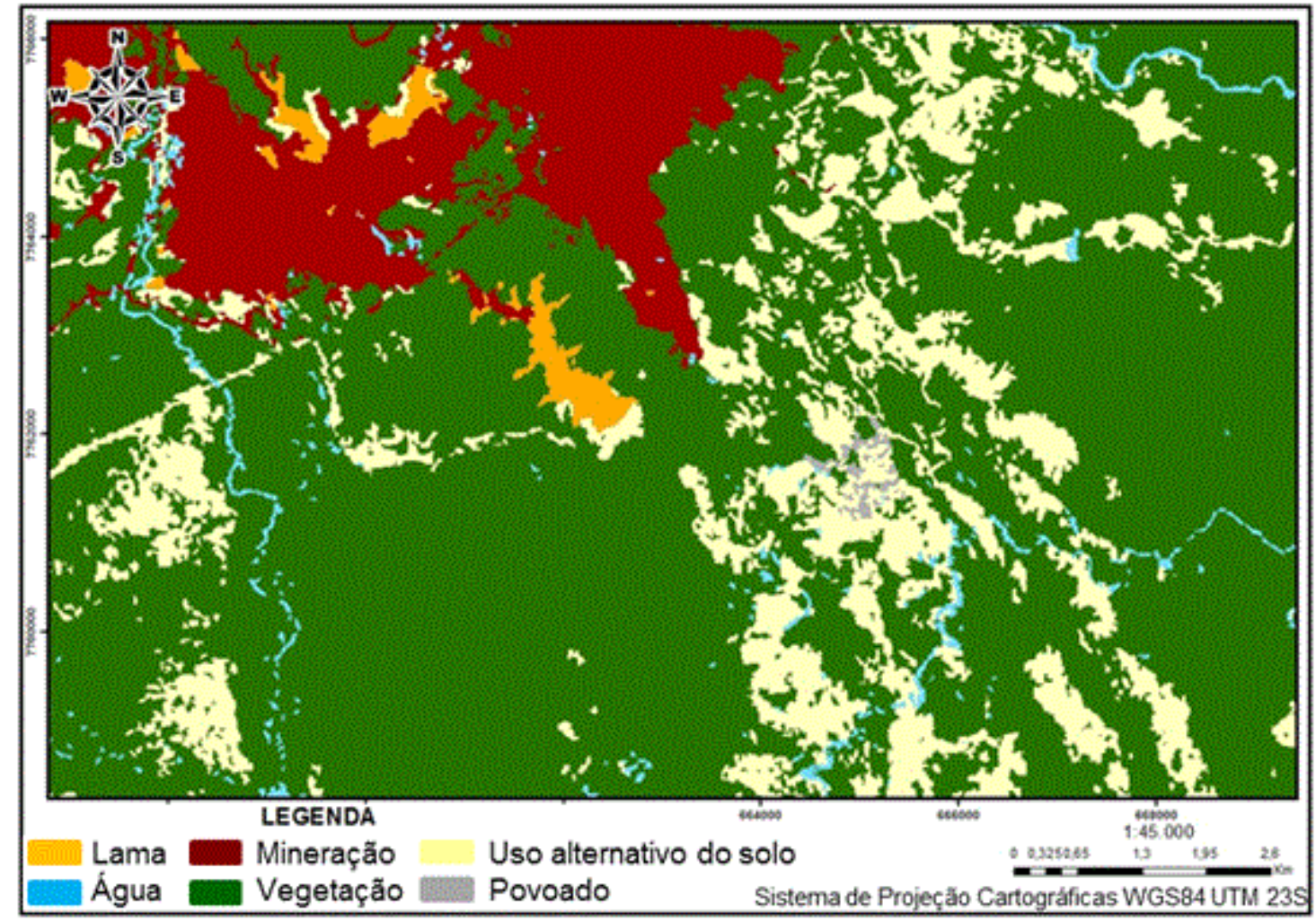

Figura 6: Classificação uso do solo pelo ArcGIS antes do desastre. Fonte: autores. 


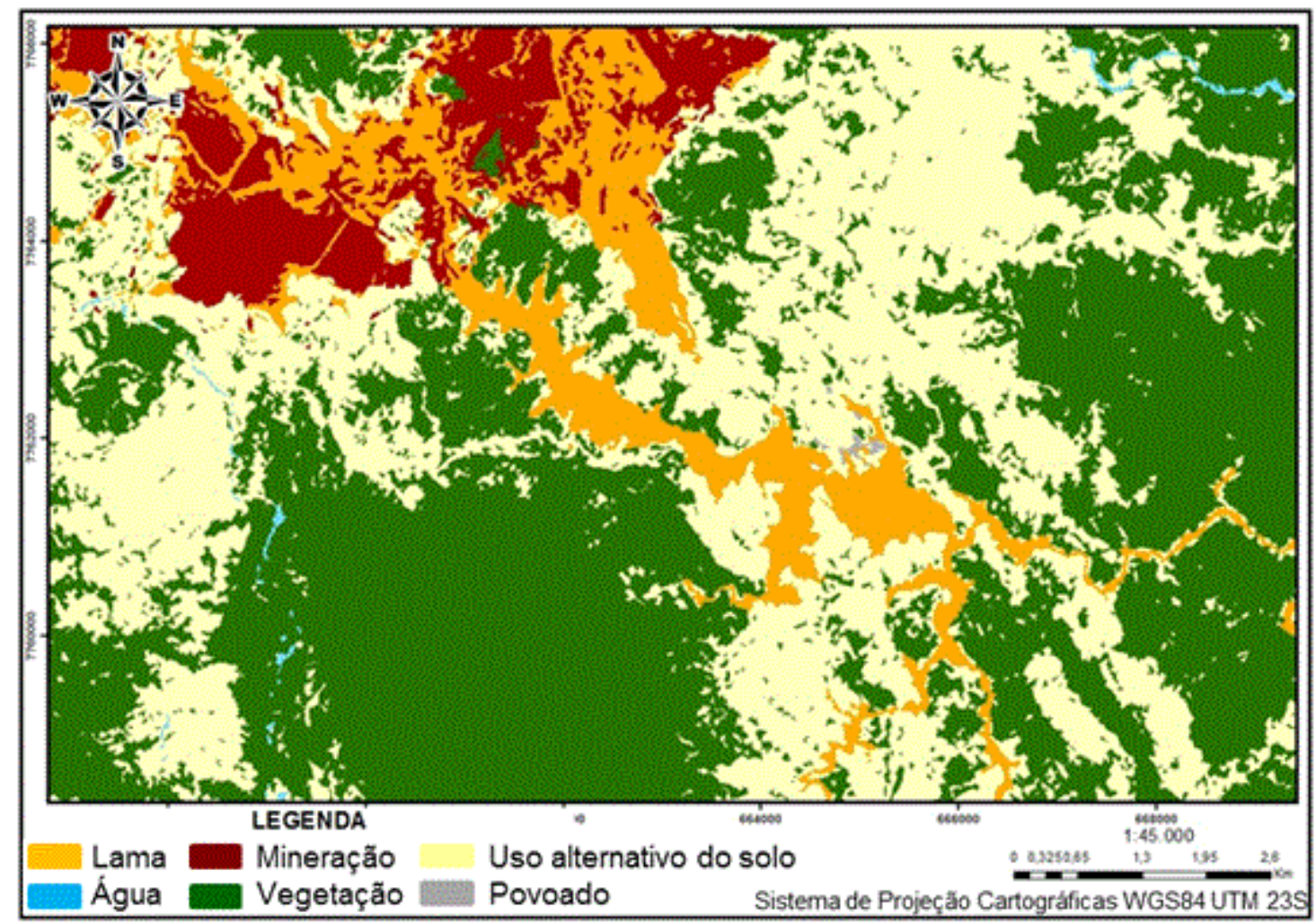

Figura 7: Classificação uso do solo pelo ArcGIS depois do desastre. Fonte: autores.

De acordo com a classificação, observa-se que o rompimento da barragem provocou uma enxurrada de lama que pavimentou as regiões próximas à barragem, devastou o subdistrito de Bento Rodrigues e atingiu os recursos hídricos que desaguam no rio Doce.

Observando as figuras 4, 5, 6, e 7 percebe-se que as classificações realizadas nos dois softwares (Spring e ArcGIS) apresentaram diferenças. Essas diferenças são destacadas nas comparações apresentadas nas tabelas 1 e 2.

Tabela 1: Comparação das áreas classificadas antes do desastre. Fonte: autores.

\begin{tabular}{l|c|c|c}
\hline Classes & ArcGIS (ha) & Spring (ha) & Diferença (\%) \\
\hline Mineração & 1322,51 & 1372,86 & 3,66 \\
Lama & 117,09 & 152,19 & 23,06 \\
Uso alternativo do solo & 1627,48 & 2616,81 & 37,8 \\
Vegetação & 8553,13 & 8431,98 & 1,41 \\
Água & 321,51 & 187,11 & 41,8 \\
Povoado & 24,41 & 23,87 & 2,21 \\
\hline
\end{tabular}

A tabela 1 mostra as diferenças das áreas classificadas através dos softwares Spring e ArcGIS antes do desastre, sendo as diferenças mais significativas encontradas na lama (23\%), no uso alternativo do solo (38\%) e na água (42\%). A tabela 2 apresenta as diferenças observadas nos resultados obtidos no Spring e no ArcGIS das classificações das imagens após o desastre, sendo as diferenças mais significativas encontradas no uso alternativo do solo (24\%), na vegetação (22\%) e na água (18\%).

Tabela 2: Comparação das áreas classificadas depois do desastre. Fonte: autores.

\begin{tabular}{l|l|l|l}
\hline Classes & ArcGIS (ha) & Spring (ha) & Diferença (\%) \\
\hline
\end{tabular}




\begin{tabular}{l|c|c|c}
\hline Mineração & 682,22 & 747,07 & 8,55 \\
Lama & 1114,34 & 1122,07 & 0,68 \\
Uso alternativo do solo & 4427,76 & 3357,51 & 24,17 \\
Vegetação & 5784,84 & 7444,5 & 22,29 \\
Água & 133,38 & 109,35 & 18,01 \\
Povoado & 4,92 & 4,88 & 0,81 \\
\hline
\end{tabular}

Essas diferenças se devem ao método utilizado na identificação das classes, que no Spring é realizada através da seleção de retângulos e no ArcGIS pela identificação de diversos pontos para cada classe. Na Figura 8, apresentam-se as áreas afetadas pela lama, bem como a distinção de cada classe.

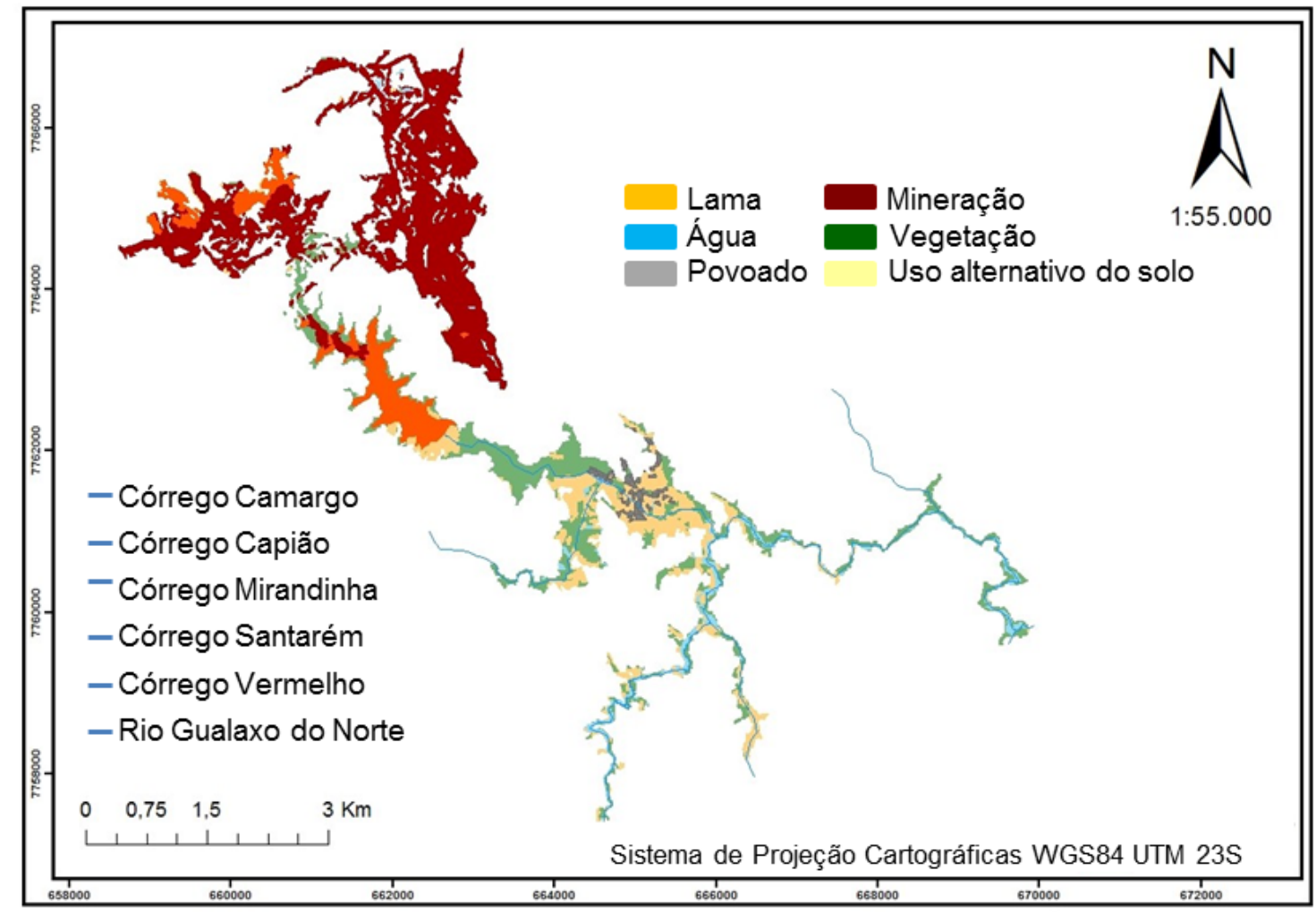

Figura 8: Áreas afetadas pela lama. Fonte: autores.

Conforme a tabela 3, a lama atingiu uma área total de aproximadamente 944 ha, sendo 249,29 ha de vegetação da Mata Atlântica, ocorrendo a destruição da vegetação ciliar, incluindo áreas de preservação permanente (APP). A vegetação ciliar é importante para a manutenção da biodiversidade e para as comunidades locais, um impacto dessa magnitude nesse ambiente provoca um desiquilíbrio no ecossistema, causando reflexos negativos na fauna e flora. No relatório apresentado pela Força Tarefa (2016), que foi um produto originado do decreto 46.892, de 20 novembro de 2015, que a instituiu para avaliação dos efeitos e desdobramentos do rompimento das Barragens de Fundão e Santarém, localizadas no Distrito de Bento Rodrigues, no Município de Mariana, foi estimado o impacto em 384,71 ha de Mata Atlântica até parte do Rio Gualaxo do Norte em direção ao Rio do Carmo. A diferença de 35\% encontrada entre o valor obtido neste estudo e o valor apresentado no relatório da Força Tarefa (2016) se deve ao fato da limitação da área de projeto, pois neste artigo foi delimitada uma área de estudo menos 
abrangente do que a apresentada no relatório da Força Tarefa (2016). Foi afetada também uma área de 125,60 ha de uso alternativo do solo, sendo a maior parte, áreas de agropecuária.

Tabela 3: Quantificação das áreas afetadas pela lama. Fonte: autores.

\begin{tabular}{lc}
\hline Classes & Área (ha) \\
\hline Lama & 93,24 \\
Água & 45,92 \\
Vegetação & 249,29 \\
Uso alternativo do solo & 125,6 \\
\hline
\end{tabular}

Em relação à água, a lama afetou 4,12 Km do córrego Santarém, 9,75 $\mathrm{Km}$ do córrego Vermelho, 3,28 Km do rio Gualaxo do Norte, 2,60 Km do córrego Mirandinha, 0,10 Km do córrego Capião e 1,95 $\mathrm{km}$ do córrego Camargo. Na área de projeto, o rompimento da barragem de rejeitos da mineradora afetou um total de $21,80 \mathrm{~km}$ de cursos d'água, contribuindo para o assoreamento dos mesmos e para a mortandade da biodiversidade aquática existente nesses recursos hídricos. Além da fauna aquática, a fauna terrestre também foi afetada prejudicando o consumo e a utilização para dessedentação dos animais.

Os dados adquiridos ao longo do trabalho, bem como as informações geradas referentes ao impacto ocasionado pela lama, podem ser acessados e manipulados facilmente no site criado para este fim. A Figura 9 representa a página disponibilizada neste endereço web.

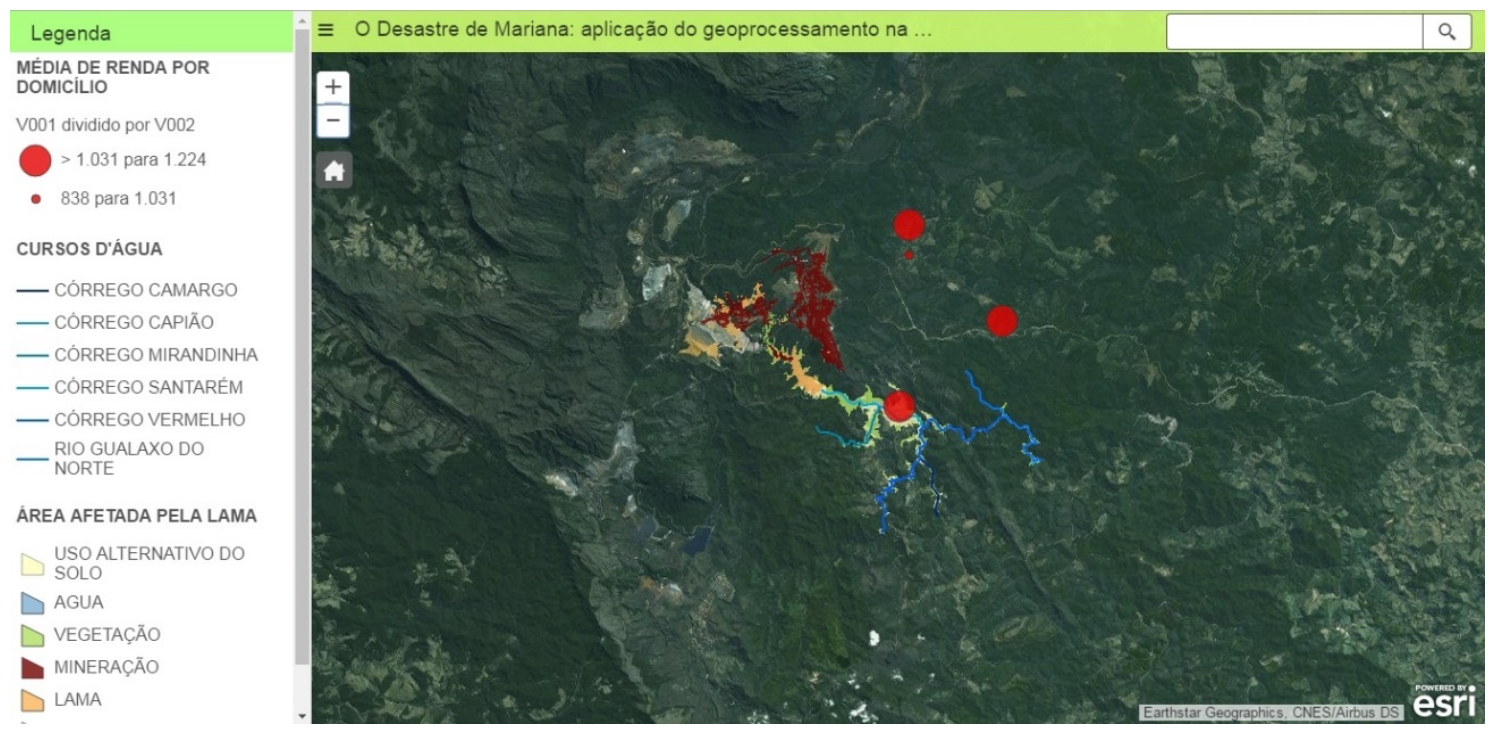

Figura 9: Publicação dos resultados no ArcGIS OnLine. Fonte: autores.

Para acessar o trabalho no ambiente online é necessário realizar um cadastro no ArcGis Online. Assim, logo após o cadastro de usuários, bastaria acessar o Link: http://www.arcgis.com/apps/SimpleViewer/index.html?appid=65614e8a36de4718814334d94f5c $171 \mathrm{c}$.

\section{CONCLUSÕES}

O uso do geoprocessamento, em especial o tratamento de imagens de satélite, permitiu quantificar a área afetada pela lama originada do rompimento da barragem de rejeitos em Bento Rodrigues. A determinação da área da lama, por sua vez, possibilitou identificar os danos 
ambientais sofridos na vegetação, nos recursos hídricos, demais atividades produtivas da população, atingindo assim o objetivo proposto na pesquisa.

Esta pesquisa se limitou a medir os danos ambientais causados pelo desastre do rompimento da barragem de rejeitos de Bento Rodrigues. Portanto resta a necessidade futura de se estudar e estimar os prejuízos relacionados à suspensão das atividades econômicas e consequentemente indicar as indenizações devidas aos prejudicados.

Neste caso, a destruição de infraestruturas, perdas de patrimônios e os prejuízos que se refletem na economia após o desastre requer a aplicação de novas abordagens e metodologias a fim de reestabelecer a ordem econômica e social, além da restauração e preservação ambiental.

\section{REFERÊNCIAS}

Aragão, R. D., \& Almeida, J. D. (2009). Avaliação espaço temporal do uso do solo na área da bacia do Rio Japaratuba-Sergipe através de imagens LANDSAT. Simpósio Brasileiro de Sensoriamento Remoto, 14, 1231-1238.

Araújo, C. B. (2006). Contribuição ao estudo do comportamento de barragens de rejeito de mineração de ferro. Universidade Federal do Rio de Janeiro, COPPE, Rio de Janeiro.

Barbosa, M. P., Moraes Neto, J. M., Fernandes, M. F., \& Silva, M. J. (2007). Estudo da degradação das terras-município de Picos-PI. Anais XIII Simpósio Brasileiro de Sensoriamento Remoto, Florianópolis, 13, 4357-4363.

Bittar Filho, C. A. (1994). Do dano moral coletivo no atual contexto jurídico brasileiro. Revista de direito do consumidor, 12, 44-62.

BRASIL, C. (1986). Resolução CONAMA No 001 de 23 de Janeiro de 1986. Dispõe Sobre as Diretrizes Gerais Para Uso e Implementação de Avaliação de Impacto Ambiental. Brasileira. Diário Oficial.

Câmara, G., Monteiro, A. M., Fucks, S. D., \& Carvalho, M. S. (2002). Análise Espacial e Geoprocessamento. Análise espacial de dados geográficos, Embrapa Cerrados Brasília, 2.

Carvalho, A., Neto, J. M. M., \& Lima, V. L. (2011). Diagnóstico da degradação da ambiental da bacia hidrográfica do açude Soledade-Paraíba. Engenharia Ambiental: Pesquisa e Tecnologia, $8(2)$.

Cerri Neto, M. (2007). Aspectos jurídicos das atividades de defesa civil. Brasília: Secretaria Nacional de Defesa Civil.

Chaves, C. J., Reis, F. A. G. V., do Carmo Giordano, L., Do Amaral, A. M. C., \& dos Santos Corrêa, C. V. (2015). Aplicação da compartimentação fisiográfica e da abordagem multitemática para elaboração de mapa geoambiental: estudo de caso nos municípios de Espírito Santo do Pinhal e Santo Antônio do Jardim (SP). Geologia USP. Série Científica, 15(2), 3-24.

Collischonn, W., \& Tucci, C. E. (1997). Análise do rompimento hipotético da barragem de Ernestina. Revista Brasileira de Recursos Hídricos, 2(2), 191-206. 
Costa Lima, G. (1999). Questão ambiental e educação: contribuições para o debate. Ambiente \& sociedade, 2(5), 135-153.

Cruz, Z. Q., Silveira, J. C., \& Ribeiro, G. P. (2009). Ensaios de segmentação e classificação digital de uma unidade de conservação com imagens CBERS utilizando o sistema SPRING. Estudo de caso: Parque Nacional da Serra dos Órgãos (PARNASO). Anais do XIV Simpósio Brasileiro de Sensoriamento Remoto. Natal: Inpe, 6853-6860.

ESRI. (2006). Environmental Systems Research Institute, ArcGIS Professional GIS for the desktop, version 9.2. Software.

Fenker, E. (2011). Impacto ambiental e dano ambiental. Artigo técnico. Recuperado em 25 de outubro, 2019, de http://www. ambientebrasil. com. br/noticias/index. php3.

Fernandes Neto, S., Silva, T. T. S., \& Dos Santos, J. S. (2015). Impactos ambientais causados pela disposição final de rejeitos provindos da mineração de quartzito na Paraíba. In Congresso Técnico Científico da Engenharia e da Agronomia CONTECC.

Força Tarefa, G. da F. (2016). Relatório: Avaliação dos efeitos e desdobramentos do rompimento da barragem de fundão em Mariana-MG.

Galo, M. D. L. B. T., Velini, E. D., Trindade, M. L. B., \& Santos, S. C. A. (2002). Uso do sensoriamento remoto orbital no monitoramento da dispersão de macrófitas nos reservatórios do complexo Tietê. Planta Daninha, (20), 7-20.

IBAMA. (2015). Impactos ambientais decorrentes do desastre envolvendo o rompimento da barragem de Fundão, em Mariana. [S.I.].

IBGE. (2001). Introdução ao processamento digital de imagens / IBGE, Primeira Divisão de Geociências do Nordeste. [S.I.: s.n.], 2001. (Coleção Ibgeana; Manuais técnicos em geociências, ISSN 0103-9598). 92 p.

INPE, Instituto Nacional de Pesquisas Espaciais. (2008). Regeemy version 0.2.43. Divisão de Processamento de Imagem - INPE. Software.

INPE, Instituto Nacional de Pesquisas Espaciais. (2016). Sistema de Processamento de Informações Georreferenciadas - Spring versão 5.4.2. DPI/INPE. 2016. [on line]. Instituto Nacional de Pesquisas Espaciais.

Jardim, W. de F. (2016). O desastre de Mariana é o retrato do Brasil. Recurado em 25 de outubro, 2019, de http://www.unicamp.br/unicamp/noticias/2015/11/23/artigo-o-desastre-demariana-e-o-retrato-do-brasil>.

Longhitano, G. A. (2010). VANTS para sensoriamento remoto: aplicabilidade na avaliação e monitoramento de impactos ambientais causados por acidentes com cargas perigosas (Doctoral dissertation, Universidade de são Paulo).

Lorenzeto, R. (2006). Mapa de localização do Município de Mariana. Recuperado em 25 de outubro, 2019, de em: https://pt.wikipedia.org/wiki/Mariana\#/media/Ficheiro:MinasGerais_Municip_Mariana.svg. 
Marcelino, E. V., Nunes, L. H., \& Kobiyama, M. (2006). Mapeamento de risco de desastres naturais do estado de Santa Catarina. Caminhos de Geografia, 7(17).

Mariana, P. M. (2016). Localização. Recuperado em 25 de outubro, 2019, de http://pmmariana.com.br/localizacao.

Maskrey, A. (Ed.). (1998). Navegando entre brumas: la aplicación de los sistemas de información geográfica al análisis de riesgos en América Latina. La Red.

Medina, J. (1994). Sistemas de información en las regiones de San Martín y Perú. Desastres y Sociedad, Lima. LA RDE/ITDG, (2), 144-150.

Meneses, P. R., \& Almeida, T. D. (2012). Introdução ao processamento de imagens de sensoriamento remoto. Universidade de Brasília, Brasília.

Meneses, P. R., \& Sano, E. E. (2012). Classificação pixel a pixel de imagens. Introdução ao processamento de imagens de sensoriamento remoto. Brasília: UnB/Cnpq, 12, 191-208.

Mora, S., \& Keipi, K. (2006). Disaster risk management in development projects: models and checklists. Bulletin of Engineering Geology and the Environment, 65(2), 155-165.

Moura, G. S. S., Barbosa, M. P., de Moura, C. S., de Araújo, A. E., de Moura, A. E. S. S., \& de Souza, M. M. P. (2005). Uso de imagens TM/Landsat-5 na avaliação da degradação ambiental e riscos a desastres ENSO, no Município de Sumé-PB. Anais do XII Simpósio brasileiro de sensoriamento remoto, Goiânia-INPE.

Pegoraro, A. J., Gubiani, J. S., \& Philips, J. W. (2013). Veículo aéreo não tripulado: uma ferramenta de auxílio na gestão pública. In Proc. Symp. Argentine of Informatics and Law, 177-187.

Petta, R. A., Castelani, R., OA Filho, F., Meyer, M., Duarte, C. R., \& Silva, N. L. (2005). Uso do Geoprocessamento (SR e SIG) em mapeamentos Ambientais de áreas de exploração de petróleo na Bacia Potiguar (RN). In Anais do 2 Congresso Brasileiro de P\&D em Petróleo e Gás.

Pimentel, T. (2016). Excesso de água em barragem provocou rompimento, diz Polícia Civil. Recuperado em 25 de outubro, 2019, de http://g1.globo.com/minas-gerais/desastreambiental-em-mariana/ noticia/2016/02/excesso-de-agua-em-barragem-provocourompimento-diz-policia-civil.html.

Pinto, W. D. P., Lima, G. B., \& Zanetti, J. B. (2015). Análise comparativa de modelos de séries temporais para modelagem e previsão de regimes de vazões médias mensais do Rio Doce, Colatina-Espírito Santo. Ciência e Natura, 37(4), 1-11.

Saito, S. M., Sausen, T. M., Lacruz, M. S. P., Madruga, R., \& Junior, E. D. R. L. (2011). Avaliação dos desastres naturais ocorridos no Rio Grande do Sul em 2008. XV Simpósio Brasileiro de Sensoriamento Remoto-SBSR.

Samarco. (2015). Sobre a Samarco. Recuperado em 25 de outubro, 2019, de http://www.samarco.com/ institucional/a-empresa. 
Sanchéz, L. E. (2015). Avaliação de impacto ambiental. [S.I.]: Oficina de Textos.

Santos, A. D., Peluzio, T. D. O., \& Saito, N. S. (2010). Spring 5.1. 2: Passo a passo: Aplicações práticas. Alegre, ES: CAUFES.

Silva, J. (2009). Mapeamento de inundações no Brasil: proposta de gestão ambiental através de um sistema de informações geográficas. IX Seminário de pós-graduação em geografia da UNESP Rio Claro.

Silva, J. P. S. (2007). Impactos ambientais causados por mineração. Revista espaço da Sophia, 8(1), 1-13.

Sousa, R. F., Barbosa, M. P., Neto, C. P. C. T., de Paula Carvalho, A., \& de Lima, A. N. (2008). Uso de geotecnologias no diagnóstico da degradação das terras do município de São João do Cariri-PB. Revista Caatinga, 21(1), 204-210.

Sousa, R., Barbosa, M., Guimarães, C., \& Carvalho, A. (2007). Avaliação das classes de cobertura vegetal e mapeamento do uso atual dos solos no município de Itaporanga-PB. Engenharia Ambiental: Pesquisa e Tecnologia, 4(1), 80-88.

USGS, United States Geological Survey. (2016). What are the best Landsat spectral bands for use in my research?. Disponível em: </https://www.usgs.gov/faqs/what-are-best-landsatspectral-bands-use-my-research?qt-news_science_products=0\#qt-

news_science_products/>. Acesso em: 30 de abril, 2016.

Zerger, A., \& Smith, D. I. (2003). Impediments to using GIS for real-time disaster decision support. Computers, environment and urban systems, 27(2), 123-141. 